\title{
Damage experiments in a cylindrical geometry
}

\author{
A. Kaul
}

Los Alamos National Laboratory, XCP-5, MS F663, Los Alamos, NM, USA

\begin{abstract}
Studying spallation damage with a cylindrical configuration allows for a natural recollection of the damaged material under proper driving conditions. Additionally, the damaged material can come to a complete rest without the application of further stopping forces. Specific areas of research include the damage initiation regime in convergent geometry, behavior of material recollected after damage, and effects of convergent geometry on the material response. Such experiments produce unique strain and shear stress states, motivating improvements in existing computational material models and increasing the predictive capabilities of codes. A LANL/VNIIEF joint experimental series has produced cylindrical aluminum failure initiation data and studied the behavior of material recollected after damage initiation and after complete failure. In addition to post-shot collection of the damaged target material for subsequent metallographic analysis, dynamic in-situ experimental diagnostics include velocimetry and transverse radial radiography. This paper will discuss the current experimental status.
\end{abstract}

\section{Experimental program}

Los Alamos National Laboratory (LANL) and The All-Russian Research Institute of Experimental Physics (VNIIEF) have developed a joint experimental series to gather data on damage in a (presumably) well-understood material, i.e. aluminum, to motivate development and validation of damage models for computational tools. Specifically, research is centered on mechanisms which control damage initiation and fracture and on the behavior of previously damaged material after cracks close. Since these single-physics experiments use a cylindrical configuration to access unique strain and shear stress states, they allow experimental validation of the multi-dimensional aspects of existing models.

The experimental driver is a VNIIEF-designed helical flux compression generator with explosive switches to control the current pulse shape and duration. The current wave form produced is a single pulse with a peak current of 4.5-10.5 mega ampere (MA), depending on the charge voltage and the generator's compression factor. A VNIIEF-designed shielding system placed between the generator and the load provides protection of the load and diagnostics from any explosive debris produced by the generator.

The shock profile in the target can be varied through changes in the impactor and target geometry and through changes in current peak and duration. In a cylindrical configuration, recollection and closure of voids can occur naturally under proper driving conditions, with no additional application of force needed to bring the system to a resting state. The chosen configuration simplifies target recovery for post-experimental metallographic analysis. In addition to the target recovery, diagnostics fielded on the experiments include free-surface velocimetry measurements using visar or photon Doppler velocimetry (PDV) and current measurements using Faraday induction loops or B-dot probes.

This is an Open Access article distributed under the terms of the Creative Commons Attribution-Noncommercial License 3.0, which permits unrestricted use, distribution, and reproduction in any noncommercial medium, provided the original work is properly cited. 


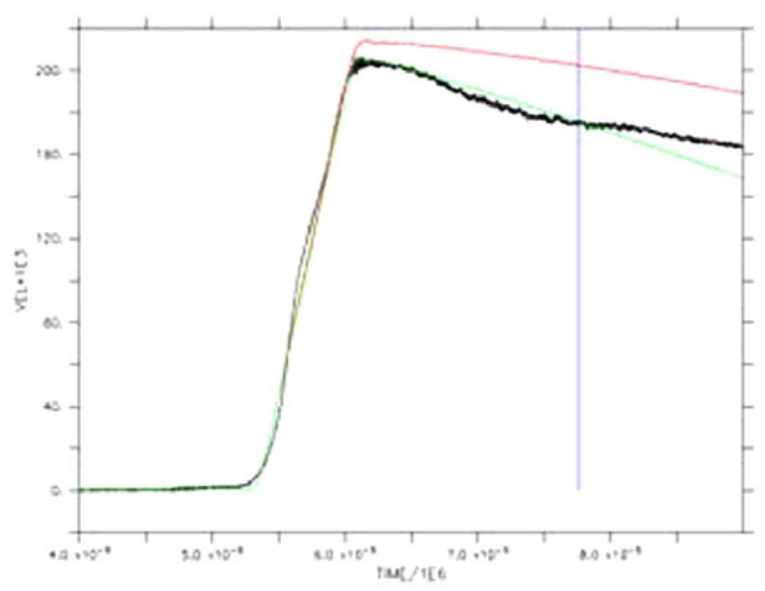

Fig. 1. Measured RD-0 velocity as a function of time in black; pre-experiment calculated velocity in red; postexperiment calculated velocity in green, target impact in blue.

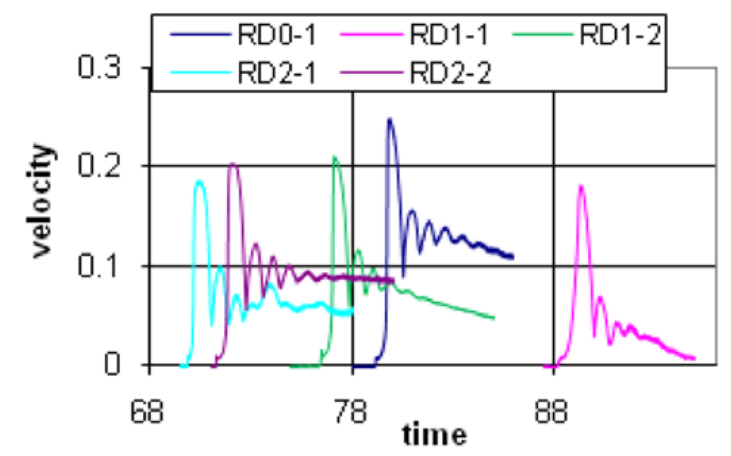

Fig. 2. Target velocimetry for RD-0, RD-1 and RD-2.

\section{Russian-damage- $0,-1$ and -2}

The first group of three experiments, designated RD-0, -1 and -2, was intended to obtain data about the initiation of voids and damage for aluminum in a cylindrical configuration. In order to compare the results with planar gas gun experiments, the impacting liner was in free flight with no driving current remaining in the system at the time of impact. The aluminum used in these loads is a specially fabricated high purity ( $\sim 98.5 \%)$ alloy. Due to its unique nature, there is very little data available to develop a set of strength parameters, so initial calculations used Aluminum 1100-O values.

The LANL-designed and fabricated experimental loads usually contain two targets in each load. Because this system used a driver new to the LANL team, the first experiment's load consisted of a bare liner on one half of the load and a target in the other half of the load. This allowed for velocimetry measurements using a visar system on the inner surface of the impacting liner for use in calibrating the design calculations. The liner velocity measurement (units of $\mathrm{m} / \mathrm{s}$ ) is shown as a function of time (units of $\mu \mathrm{s}$ ) in black in Fig. 1. The blue vertical liner indicates impact with the target, determined by integration of the velocity signal. The red line is the expected liner velocity from pre-experiment calculations. Clearly, the liner did not behave as expected, but rather as if it were much stronger and less susceptible to dynamic strength hardening. Post-experiment parameter searches resulted in the calculated liner velocity shown in green, which matches the measured peak and impact velocities.

For the one target in the first experiment and the two targets in each of the other two experiments, the visar system also measured the inner free-surface velocities. The recorded velocities (units of $\mathrm{km} / \mathrm{s}$ ) are shown as a function of time (units of $\mu \mathrm{s}$ ) in Fig. 2. Table 1 provides a summary of the three experiments, including target size (in mm), peak currents (in MA), RD-0 measured liner impact velocity 
New Models and Hydrocodes for Shock Wave Processes in Condensed Matter

Table 1. Summary of RD-0, RD-1 and RD-2 measurements and calculations.

\begin{tabular}{lccccc}
\hline & RD-0 & RD-1 T1 & RD-1 T2 & RD-2 T1 & RD-2 T2 \\
\hline R, outer & 44 & 43 & 45 & 46 & 46 \\
\hline R, inner & 24 & 23 & 25 & 36 & 26 \\
\hline I, peak & 5.0 & 4.8 & 4.8 & 4.8 & 4.8 \\
\hline Vel, impact & 176 & & & & 158 \\
\hline Vel, impact (calculated) & 176 & 125 & 146 & 158 & 203 \\
\hline Vel, peak free surface & 248 & 182 & 210 & 187 & 218.8 \\
\hline Vel, peak fs (calculated) & 247.3 & 162.3 & 205.1 & 188.9 & Crack \\
\hline Damage State & Full & Voids & Crack & Crack & Crack
\end{tabular}

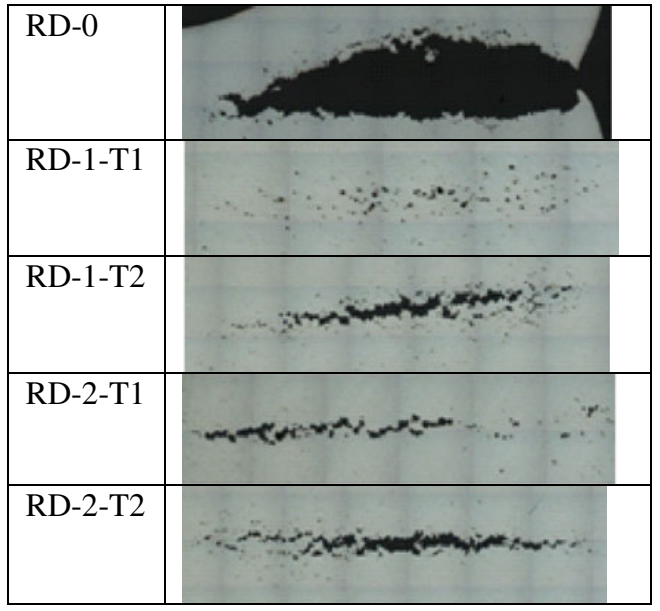

Fig. 3. Results of target metallography.

(in $\mathrm{m} / \mathrm{s}$ ), calculated liner impact velocity (in $\mathrm{m} / \mathrm{s}$ ), measured and calculated target peak free surface velocity (in $\mathrm{m} / \mathrm{s}$ ) and damage state seen in metallographic analysis. The RD-0 target free surface velocimetry was used to adjust target strength parameters to account for the unique material. As can be seen from the table, the resulting calculations for target peak free surface velocity in experiments RD-1 and RD-2 are reasonably close to the measured values. As a comparison, damage initiation in planar flyer plates occurs in the range between 198 and $210 \mathrm{~m} / \mathrm{s}$ for this material. At $198 \mathrm{~m} / \mathrm{s}$, only a few voids were visible. At $210 \mathrm{~m} / \mathrm{s}$, a full crack had formed.

Figure 3 shows images from metallographic analysis of the recovered targets. RD-0 exhibits a complete failure state in the center of the target. RD-1-T1 exhibits a state of incipient failure, with only void formation visible. RD-1-T2 shows a complete crack in the center, with only voids on the edges. RD-2-T1 exhibits a state with a less complete crack and more voids, while RD-2-T2 has a more complete crack with voids still visible.

Faraday induction loop current (in MA) as a function of time (in s) measurements for RD-0 are shown in Fig. 4. The half-height pulse duration is $8 \mu \mathrm{s}$, while the peak currents in the 3 traces vary between 4.88 and 4.91 MA.

\section{Russian-damage-3, -4 and -5}

The second group of three experiments, designated RD-3, -4 and -5, was intended to obtain data about material recollected after the initiation of voids and damage. To achieve recollection, the driving current remained in the system at the time of initial liner impact and was removed only after the second liner impact which provided the recollection force. Removing the current allowed the assembly to 


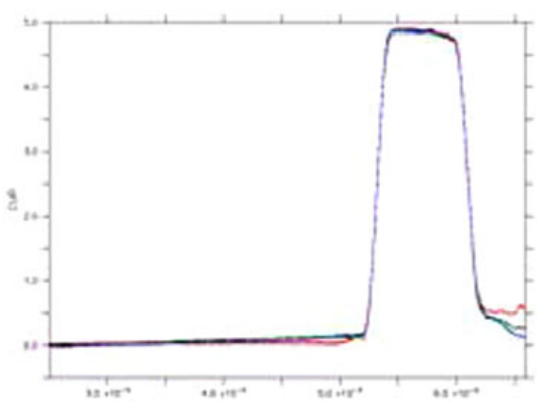

Fig. 4. Current measurement for RD-0.

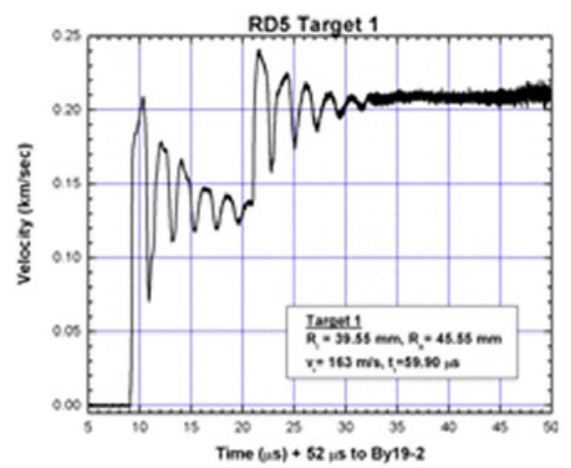

Fig. 5. RD-5 target 1 inner free surface velocity.

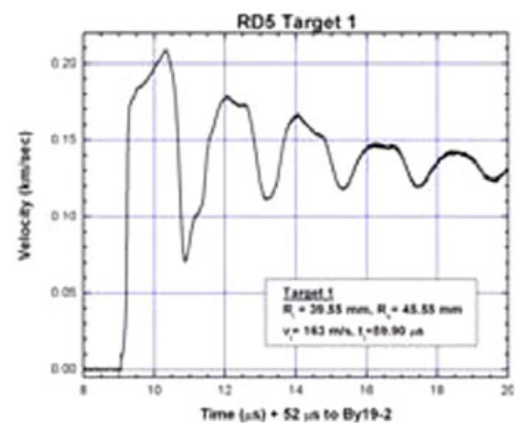

Fig. 6. Expansion of early time RD-5-T1 free surface velocity.

come to rest with no further input forces. To address the issues raised in the first experiments about the motion of the liner prior to impact, a velocimetry window was placed in the glide plane between the two targets.

Figures 5-8 are the visar measurements of the target inner free surface velocity for the two targets in RD-5. Figures 5 and 7 show the full signal for targets 1 and 2, respectively, while figures 6 and 8 are enlargements of the early-time signals. The signals for both targets show shock breakout, incipient spall, ringing, then a second shock-free push from the liner, followed by more ringing.

In addition to the targets, visar was used to measure the liner inner free surface velocity. These measurements are shown in Fig. 9, with expansion of the early signals in Fig. 10. The dots on the traces in Fig. 10 indicate liner impact with the two targets for each experiment. As the wave reflected from the edge of the window reaches the visar at a slightly later time, the velocity signature exhibits an abrupt change as the liner begins to accelerate through the window. The ringing corresponds to wave 


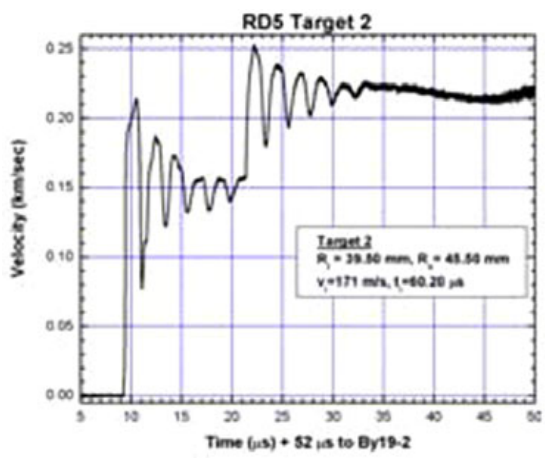

Fig. 7. RD-5 target 2 inner free surface velocity.

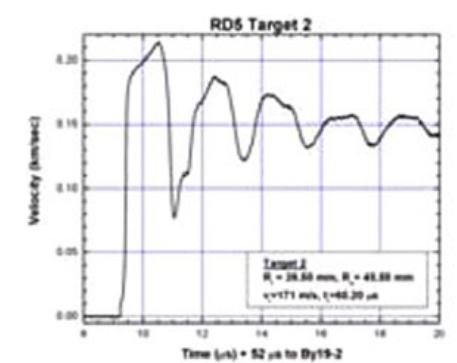

Fig. 8. Expansion of early time RD-5-T2 free surface velocity.

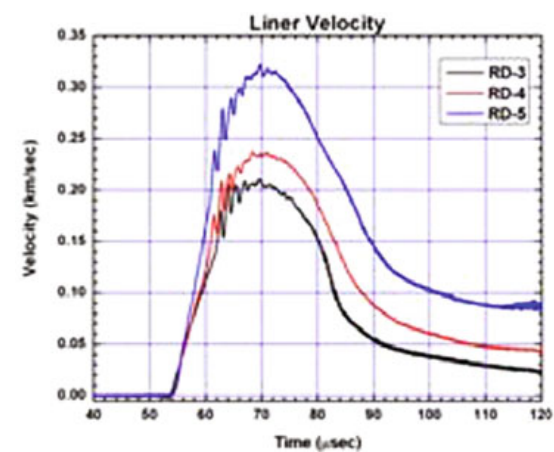

Fig. 9. Liner inner free surface velocities.

speeds across the window opening. The liner velocity then drops off as the liner attempts to push a fairly thick piece of copper and the current is removed.

Table 2 provides a summary of the three experiments, including target size (in $\mathrm{mm}$ ), measured and calculated liner impact time (in $\mu \mathrm{s}$ ) and velocity (in $\mathrm{m} / \mathrm{s}$ ), measured and calculated target breakout time (in $\mu \mathrm{s}$ ) and peak free surface velocity (in $\mathrm{m} / \mathrm{s}$ ), and estimated damage state. The calculations are based on the measured currents and used the strength parameters calculated from the RD-0 results. As can be seen from the table, the resulting calculations are reasonably close to the measured values. The impact velocities attained in RD-3 and RD-4 were insufficient to produce voids in the material. In addition, the visar signal in RD-3-T2 was affected by insufficient shielding of the fibers. The half-height pulse duration for the current was $22-24 \mu \mathrm{s}$, while the peak current ranged from 5.6 MA for RD-3 to 7.2 MA for RD-5. 
EPJ Web of Conferences

Table 2. Summary of RD-3, RD-4 and RD-5.

\begin{tabular}{lcccccc}
\hline & RD-3 T1 & RD-3 T2 & RD-4 T1 & RD-4 T2 & RD-5 T1 & RD-5 T2 \\
\hline R, outer & 45.45 & 45.40 & 45.65 & 45.60 & 45.55 & 45.50 \\
\hline R, inner & 39.45 & 39.40 & 39.65 & 39.60 & 39.55 & 39.50 \\
\hline Impact time & 61.52 & 61.88 & 59.79 & 60.20 & 59.90 & 60.20 \\
\hline Vel, impact & 137.0 & 146.0 & 122.0 & 130.0 & 165.0 & 173.0 \\
\hline Vel, impact (calculated) & 133.0 & 138.7 & 120.3 & 127.2 & 162.4 & 170.8 \\
\hline Breakout time & 62.30 & 62.75 & 61.17 & 61.53 & 61.34 & 61.54 \\
\hline t, brk (calc) & 63.00 & 63.34 & 61.60 & 62.0 & 63.58 & 61.88 \\
\hline Vel, peak free surface & 156.0 & 206.0 & 162.0 & 163.0 & 209.0 & 216.4 \\
\hline Vel, peak fs (calculated) & 170.6 & 176.8 & 161.0 & 169.4 & 216.4 & 225.9 \\
\hline Damage State & None & None & None & None & Voids & Voids \\
\hline
\end{tabular}

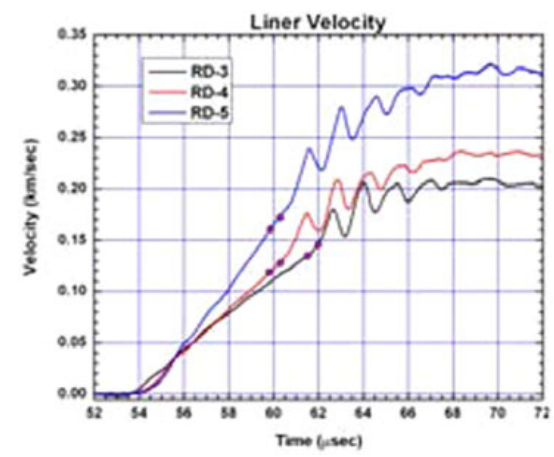

Fig. 10. Expansion of early time liner free surface velocities.

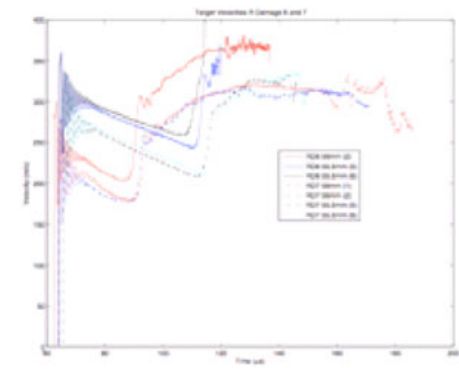

Fig. 11. Target velocimetry for RD-6 and RD-7.

\section{Russian-damage- 6 and -7}

The third group of experiments, RD-6 and -7, were designed to obtain data about material recollected after complete fracture. Achieving complete fracture required a higher initial impact velocity than the previous set of experiments and recollection of the spalled layer required a longer driving time. Therefore, the liner and target were enlarged, a thinner liner was used, the liner to target distance was increased, and PDV velocimetry was used to decrease the size of the central measuring unit, while allowing multiple measurements of each surface.

Velocimetry results for the targets are shown in Fig. 11, with an enlargement of the early time in Fig. 12. All of the signals show typical spallation signatures followed by the second shock-free loading. The ring period of each signal and its corresponding layer thickness are listed in Table 3. The initial target thickness was $6 \mathrm{~mm}$, indicating the formation of a surface inside the target. 
Table 3. Ring period and layer thickness for RD-6 and RD-7.

\begin{tabular}{lcc}
\hline Target \#-(mm) & Period $(\mu \mathrm{s})$ & Layer $(\mathrm{mm})$ \\
\hline $6-56$ & 1.134 & 3.67 \\
\hline $6-55.5$ & 1.152 & 3.73 \\
& 1.145 & 3.71 \\
\hline $7-56$ & 1.122 & 3.64 \\
& 1.128 & 3.65 \\
\hline $7-55.5$ & 1.150 & 3.73 \\
& 1.150 & 3.73 \\
\hline
\end{tabular}

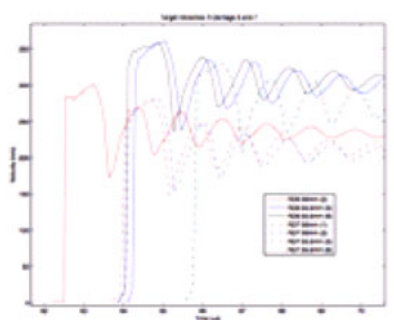

Fig. 12. Expansion of early time RD-6 and RD-7 velocimetry.

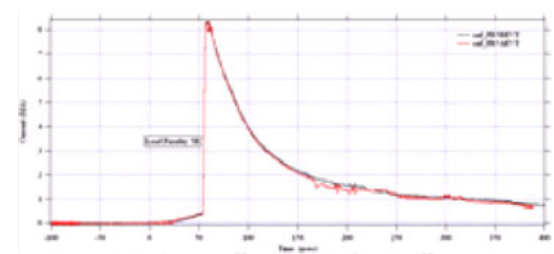

Fig. 13. RD-7 current measurement.

The targets in these two experiments collapsed to the center rather than stopping, as in earlier experiments. The current measurements, as shown in Fig. 13 for RD-7, give an explanation. The lingering current tail continues to push the liner into the target until quite late times.

Target metallographic analysis for RD-3 through -7 continues. Initial results indicate a region of intense deformation, but it is unclear how many voids or cracks still remain in the material after recollection.

\section{Conclusions}

The experimental series presented has obtained data on damage in aluminum using a cylindrical configuration. Both damage initiation and post-damage recollection have been studied. The current tail in the last two experiments was enough to overcome the mechanical strength of the assembly, pushing it to collapse on center. A different switch will be used in the two remaining experiments of the series to allow the target to stop before impact with the central measuring unit.

Metallographic analysis of the recovered targets and numerical 1-D and 2-D calculations continue. The results of the 1-D calculations presented are consistent with the measured data. 